\title{
Current Trend of Antibiotic Resistance in Lower Respiratory Tract Infections (LRTIs): An Experience in a Teaching Hospital in Bangladesh
}

\author{
Borkot Ullah $^{1}$, Sohel Ahmed ${ }^{2}$, Masum Shahariar' ${ }^{\text {and Saquiba Yesmine }}{ }^{1}$ \\ ${ }^{1}$ Department of Pharmacy, Jahangirnagar University, Savar, Dhaka-1342 \\ ${ }^{2}$ Department of Biochemistry and Molecular Biology, Jahangirnagar University, Savar, Dhaka
}

Received: December 20, 2015; Accepted: January 27, 2016; Published (Web): February 17, 2016

\begin{abstract}
Lower respiratory tract infections (LTRIs) are responsible for the vital causes of morbidity and mortality in all ages. In recent years, antibiotic resistance among respiratory pathogens is increasing emergently. The present study aimed to find out the bacterial etiology of LRTIs in Bangladesh and to update the clinicians about the current scenario of antibiotic resistance in lower respiratory tract infections. A total 64 bacterial pathogens from 100 patients with LRTIs were isolated from sputum specimens from a teaching hospital in Savar, Bangladesh between July- 2014 to June- 2015. The sputum specimens received within this year were subjected to analysis with microscopy, culture and susceptibility testing using conventional bacteriologic methods. Out of 100 processed sputum samples 64 (64\%) cases had established bacterial etiology. Staphylococcus aureus (57.81\%) was found to be the predominant organism in LRTIs followed by Streptococcus pneumonia (25\%), Klebsiella and Pseudomonas species. Males (64.06\%) were found to be more susceptible to LRTIs than that of females (35.93\%). In antimicrobial susceptibility testing, grampositive organisms showed maximum sensitivity to imipenem $(94.6 \%)$, meropenem $(97.3 \%)$ and cefotaxime (75\%). The resistance pattern varied for different organisms. Staphylococcus aureus isolates were mostly resistant to amoxicillin and ceftazidime (89.2\%), whereas, Streptococcus pneumonia was to ceftazidime, amoxicillin and cotrimoxazole $(81.2 \%)$. In case of gram negative isolates, Klebsiella sp. was mostly resistant to ceftriaxone, ceftazidime and amoxicillin (100\%). Escherichia coli were resistant to amoxicillin, cotrimoxazole and vancomycin (100\%). Therefore, appropriate identification of the causative organisms and their antibiotic resistance is crucial for right choice of antibiotic therapy in LRTIs.
\end{abstract}

Key words: LRTI, bacterial isolates, GPC, GNB, antibiotic resistance, antimicrobial susceptibility

\section{Introduction}

Respiratory tract infections are the most common infections of human being (Ndip, 2008). Lower Respiratory Tract Infections (LRTIs) are among the frequently reported infectious diseases of human in worldwide (Carroll, 2002; Mahon, 2007). LRTIs are responsible for $4.4 \%$ of all hospital admissions and $6 \%$ of all general practitioner consultations (Panda et al., 2012).

LRTI remains an important cause of morbidity and mortality worldwide; proper identification of the likely pathogens and their antibiotic susceptibility pattern can aid in the choice of antibiotic therapy and improve the outcome. Since bacterial etiology may vary in different geographical regions and even over time in the same location and population, routine surveillance of microbial etiology of LRTI is important (Tripathi, 2014). The incidence and associated mortality due to LRTI can be influenced by several factors including characteristics of the population at risk, standard of the healthcare facilities available, immunosuppressive drugs, inappropriate antibiotic therapy, distribution of causative agents, and prevalence of antimicrobial resistance (Gagneja, 2011). The causative agents of LRTIs vary from area to area and in their antibiotic susceptibility profile. Gram-positive bacteria such as

Correspondence to: Saquiba Yesmine, E-mail: s.yesmine@juniv.edu 
Staphylococcus aureus, Streptococcus pneumoniae, etc. and gram-negative bacteria e.g. Pseudomonas spp., Escherichia coli, Klebsiella spp. were identified in the LRTI patients (Mishra, 2012). Unnecessary and inappropriate initial antibiotic therapy is a potentially modifiable factor that has been associated with increased mortality in patients with serious infections (Kollef, 2011).

The choice of antimicrobial therapy for bacterial LRTIs is relatively straight forward when the etiologic agents and their antibiotic susceptibility patterns are known. However, the clinical presentation is usually not specific enough to make a firm etiologic diagnosis whether in the community or hospital setting (Shah et al., 2010). In almost all cases, eradication of causative agents requires initiation of antimicrobial therapy before obtaining culture report; however, during the last few years, the increase in antibiotic resistance has compromised the selection of empirical treatment (Jonaidi, 2009) and how to choose an effective antimicrobial agent is a new challenge to the clinicians, as the composition and the resistance to antimicrobial agents of infection pathogens was changing frequently. This trend is presumably due to the empirical administration of antibacterial therapy even before the availability of the culture results (Ahmed, 2013). Various other factors also contribute to the emergence of resistance such as irrational use of antibiotics, transmission of resistant bacteria from patient to patient and from healthcare practitioners to patients and vice versa (WHO, 2012; Mahmoud and Balkhy, 2012).

Nowadays, antibiotic resistance exerted by microorganisms against antibiotics is considered as a serious issue by global medicinal and research community (WHO, 2012; Mahmoud and Balkhy, 2012). Therefore, the clinicians and microbiologists worldwide are focusing on knowledge and strategies to limit the development of antimicrobial resistance. Current knowledge of bacterial etiology and microbial susceptibility would help reduce the indiscriminate antibiotic use and result in better therapeutic outcome and decrease in development of resistance. Evidence showed that bacteriological more effective antibiotics can reduce overall management costs, particularly with respect to consequential morbidity and hospital admission.
This study was conducted with the aim of tracking resistance rate among the causative agents of LRTI and to determine the current antibiotic resistant pattern of these bacterial isolates. The knowledge of likely prevalent strains along with their antimicrobial resistance pattern will help in better management of patients and framing the antibiotic policy.

\section{Materials and Methods}

Study design: This was a retrospective study of bacteria isolation pattern of respiratory tract samples for all ages and gender and to determine their antibiotic susceptibility pattern in a teaching hospital in Savar, Dhaka. Samples taken for processing was sputum. The duration of the study was from June 2014 to July 2015.

Specimen collection: In the present study, a total of 100 sputum samples were collected from patients who attended various wards of the hospital. All patients had clinical evidence of respiratory tract infections and a single positive culture per patient was included in the analysis.

The specimens were collected aseptically from 100 patients. Every patient were instructed on how to collect the sputum samples and taken to the microbiology lab immediately for analysis. The sputum samples were collected into well-labeled sterile, glass bottles with screw cap tops.

Specimen culture: The sputum was decontaminated by the standard alkaline decontamination method (WHO, 2012). The digested sputum samples were cultured on chocolate agar, sheep blood agar (5\%), and MacConkey agar (Oxoid, UK) plates. On the Chocolate agar, bacitracin (10 units) and optochin disks $(5 \mu \mathrm{g})$ (Oxoid, UK) were placed at secondary inoculation to screen $S$. pnemoniae. The chocolate agar plates were incubated in a incubator $\left(5-10 \% \mathrm{CO}_{2}\right)$ at $37{ }^{\circ} \mathrm{C}$ for $24-$ 48 hours while blood agar and MacConkey agar were incubated in an aerobic atmosphere at $37^{\circ} \mathrm{C}$ for 24 hours. Suspicious colonies were then subcultured on suitable solid culture media for purification and thereafter preserved on appropriate agar slants and stored in a refrigerator $\left(4^{\circ} \mathrm{C}\right)$ for subsequent analysis.

Identification of isolated organisms: The identification of isolates were accomplished using the standard microbiological techniques, which involved 
morphological colony studies, gram staining reactions, and a battery of biochemical tests as required (Cheesbrough, 2000). A colony count of $\geq 104 \mathrm{CFU} / \mathrm{ml}$ was considered to be significant for sputum (Paganin, 2004) while for other specimens $\geq 105 \mathrm{CFU} / \mathrm{ml}$ was suggestive for infection (CLSI. 2012).

Antibiotic susceptibility testing: The antimicrobial susceptibility of the test strains of fourteen antibiotics drugs was accomplished using the Kirby-Bauer disk diffusion method (Bauer, 1966) as per the Clinical Laboratory Standards Institute guidelines (CLSI, 2012). Antibiotic discs were purchased from commercial suppliers of the manufacturers Becton Dickinson and Company, USA; Oxoid Ltd., UK; Mast Diagnostics, UK. The susceptibility patterns of the bacterial pathogens were determined towards the following antimicrobial agents: azithromycin (AZT), amoxicillin (AMX), imipenem (IPM), cefotaxime (CFT), ceftriaxone (CTX), cefuroxime (CEF), ciprofloxacin (CPX), cloxacilin (CLX), gentamycin (GTM), meropenem (MPM), vancomycin (VCM), ceftazidime (CFZ), cotrimoxazole (CMX) and cefixime (CFM). A lawn of test pathogen was prepared by smoothly spreading $100 \mu \mathrm{l}$ inoculums with the help of a sterilized spreader onto the entire surface of the agar plate. The plates were dried before applying antibiotic disc. Then, some commercially available antibiotic discs were gently and firmly placed on the agar plates, which then left at room temperature for $1 \mathrm{~h}$ to allow diffusion of the antibiotics into the agar medium. The plates were incubated at $37^{\circ} \mathrm{C}$ for $24 \mathrm{~h}$, and the diameters of zone of inhibition were compared with those of the standard isolates (E. coli, ATCC 25922; P. aeruginosa, ATCC 27853; S. aureus, ATCC 25923; S. pneumonia, ATCC 49619; K. pneumonia, ATCC 700603) to determine the sensitivity or resistance based on Clinical and Laboratory Standards Institute guidelines, Wayne, Pennsylvania (CLSI. 2012).

\section{Ethical considerations}

The research was conducted following the general principles of WMA declaration of Helsinki (section 12). This research was logistically supported by the Department of Pharmacy, Jahangirnagar University and Enam Medical College and Hospital Savar, Dhaka.
Confidentiality of the patients' information was maintained.

Statistical analysis: proportions of respiratory pathogens isolated strains resistant to each of the antibiotic were measured for each of the bacterial pathogens separately. Data were analyzed using Microsoft Excel 2007.

\section{Results}

Prevalence of significantgrowth of isolatesin patient with LRTIs: In this study, a total of 100 samples were analyzed consisting of $58 \%$ in male and $42 \%$ in female (Table 1). A total of $64 \%$ isolates were recorded as pathogenic while normal flora recorded was $36 \%$.

Table 1. Prevalence of significant pathogens among the subjects with LRTIs isolated from sputum from July 2014-June 2015.

\begin{tabular}{ccc}
\hline Sex & No. tested $(\%)$ & No. tositive $(\%)$ \\
\hline Males & $58(58)$ & $41(70.7)$ \\
Females & $42(42)$ & $23(54.8)$ \\
Total & $100(100)$ & $64(64)$ \\
\hline
\end{tabular}

Note: $\mathrm{EMCH}=$ Enam medical college $\&$ hospital

Distribution of the various pathogenic bacteria isolates according to gender: Staphylococcus aureas had the highest occurrence rate with $57.81 \%$, followed by Streptococcus pneumonia 25\%, the lowest rate was recorded by $E$. coli $3.12 \%$, and details of other isolates are shown in figure 1.

Age related distribution of pathogenic bacteria isolates in LRTIs: The most of pathogens were found among patients in age range 21-30 years with a rate of $64.0 \%$, closely followed by age range $41-50$ years with $63.6 \%$ the lowest rate was recorded in age range 81-90 years with $50.0 \%$, details of the results are shown in table 2 .

Distribution of bacterial isolates in relation to gram reactions: Among the total 64 bacterial isolates, $S$. aureus $(57.81 \%)$ was found to be the most predominant organism which was isolated, followed by S. pneumonia (25\%) and Pseudomonas sp. (3.12\%), (Table 3). The gram positive cocci constituted (89.6\%) while gram negative bacilli were $(10.93 \%)$ of the total isolates (Table 3). 


\section{Antimicrobial sensitivity testing of pathogens}

Table 4 summarizes the susceptibility testing results obtained with the 5 most frequent bacterial species. S. aureus was the most prevalent bacteria. The spectrum rank order of the antimicrobial agents against S. aureus in terms of percentage of susceptibility was: meropenem $(97.3 \%)>$ imipenem $(94.6 \%)>$ gentamycin $(78.3 \%)>$ azithromycin $(72.9 \%)>$ cefuroxime $(72.9 \%)$ $>$ ciprofloxacin $(67.6 \%)$. On the other hand, S. aureas was mostly resistant to amoxicillin (89.2\%) and ceftazidime (89.2\%), cloxacilin (48.2\%). S. pneumonia had a susceptibility profile of $100 \%$ against imipenem, $87.5 \%$ meropenem, $87.5 \%$ gentamycin and $75 \%$ cefotaxime. This pathogen showed extremely high rates of resistance $(81.2 \%)$ against ceftazidime, amoxicillin, cotrimoxazole. Klebsiella sp. showed high susceptibility $100 \%$ against azithromycin, cefixime and imipenem. E. coli was resistant to amoxicillin, cotrimoxazole and vancomycin $(100 \%)$.

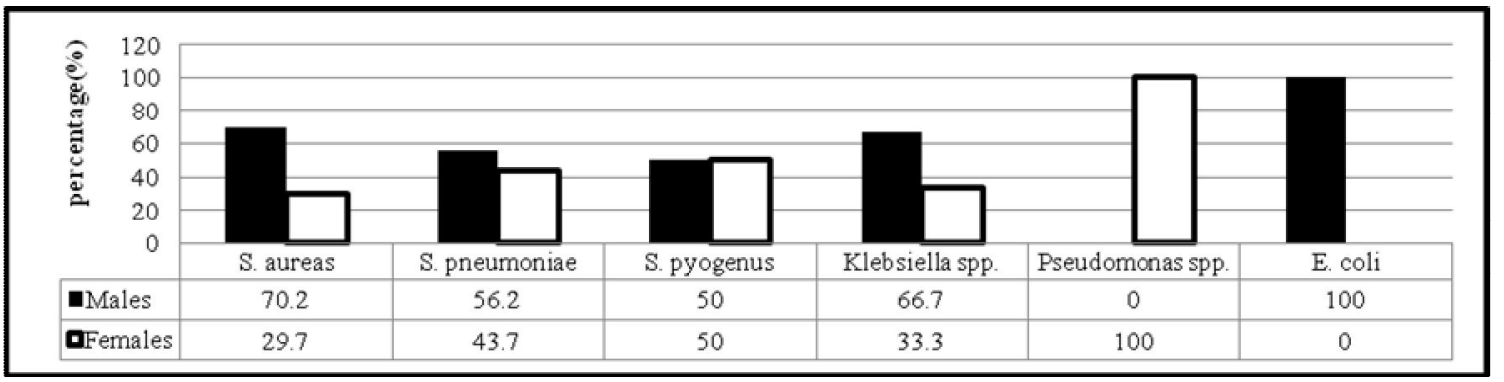

Figure 1. Distribution of pathogenic bacteria in relation to gender isolated from sputum at EMCH in Savar, Dhaka, Bangladesh from July 2014- June 2015.

Table 2. Distribution of bacterial species obtained from different age groups isolated from sputum at EMCH in Savar, Dhaka, Bangladesh from July 2014- June 2015.

\begin{tabular}{lccccccccc}
\hline \multirow{2}{*}{ Bacterial species } & \multicolumn{1}{c}{ Age groups (year) distribution } \\
\cline { 2 - 10 } & $11-20$ & $21-30$ & $31-40$ & $41-50$ & $51-60$ & $61-70$ & $71-80$ & $81-90$ & Total no. (\%) \\
\hline S. aureas & 2 & 9 & 7 & 8 & 4 & 6 & 0 & 1 & $37(57.81)$ \\
S. pneumoniae & 1 & 2 & 1 & 5 & 2 & 2 & 3 & 0 & $16(25.00)$ \\
S. pyogenus & 0 & 3 & 0 & 0 & 0 & 1 & 0 & 0 & $4(6.25)$ \\
Klebsiella spp. & 0 & 1 & 1 & 0 & 1 & 0 & 0 & 0 & $3(4.68)$ \\
Pseudomonas spp. & 0 & 0 & 1 & 0 & 1 & 0 & 0 & 0 & $2(3.12)$ \\
E. coli & 0 & 1 & 0 & 1 & 0 & 0 & 0 & 0 & $2(3.12)$ \\
Total no. (\%) & $3(4.68)$ & 16 & 10 & 14 & 8 & $9(14.06)$ & $3(4.68)$ & 1 & $64(100)$ \\
& & $(25.00)$ & $(15.62)$ & $(21.87)$ & $(12.50)$ & & & $(1.56)$ & \\
\hline
\end{tabular}

Note: $\mathrm{EMCH}=$ Enam medical college \& hospital

Table 3. Distribution of total bacterial isolates according to gram-negative and gram-positive bacteria isolated from sputum at EMCH in Savar, Dhaka, Bangladesh from July 2014- June 2015.

\begin{tabular}{llll}
\hline Bacteria & Bacterial species & Isolate no. & Isolate $(\%)$ \\
\hline & S. aureas & 37 & 57.81 \\
Gram-positive & S. pneumoniae & 16 & 25 \\
$\mathrm{n}=57(89.06 \%)$ & S. pyogenus & 4 & 6.25 \\
Gram-negative & Klebsiella spp. & 3 & 4.68 \\
$\mathrm{n}=7(10.93 \%)$ & Pseudomonas spp. & 2 & 3.12 \\
& E. coli & 2 & 3.12 \\
\hline
\end{tabular}

Note: $\mathrm{EMCH}=$ Enam medical college $\&$ hospital 
Table 4. Prevalence of resistance to recommended antibiotics in organisms causing LRTIs isolated from sputum at EMCH in Savar, Dhaka, Bangladesh from July 2014- June 2015.

\begin{tabular}{|c|c|c|c|c|c|c|}
\hline Antibiotics & $\begin{array}{c}\text { S. aureas } \\
\text { no. }(\%) \text {, } \\
\mathrm{n}=37\end{array}$ & $\begin{array}{c}\text { S. pneumoniae } \\
\text { no. }(\%), \mathrm{n}=16\end{array}$ & $\begin{array}{l}\text { S. pyogenus } \\
\text { no. }(\%), \mathrm{n}=4\end{array}$ & $\begin{array}{c}\text { Klebsiella sp. } \\
\text { no. }(\%), \mathrm{n}=3\end{array}$ & $\begin{array}{c}\text { Pseudomons sp. } \\
\text { no. }(\%), \mathrm{n}=2\end{array}$ & $\begin{array}{c}\text { E. coli } \\
\text { no. }(\%), \\
\mathrm{n}=2\end{array}$ \\
\hline Azithromycin & $10(27)$ & $7(43.7)$ & $1(25)$ & $0(0)$ & $0(0)$ & $1(50)$ \\
\hline Ciprofloxacin & $12(32.4)$ & $6(37.5)$ & $2(50)$ & $0(0)$ & $1(50)$ & $0(0)$ \\
\hline Ceftriaxone & $20(54)$ & $6(37.5)$ & $3(75)$ & $3(100)$ & $2(100)$ & $1(50)$ \\
\hline Ceftazidime & $33(89.2)$ & $13(81.2)$ & $4(100)$ & $3(100)$ & $2(100)$ & $1(50)$ \\
\hline Cefixime & $22(59.4)$ & $7(43.7)$ & $2(50)$ & $0(0)$ & $2(100)$ & $0(0)$ \\
\hline Imipenem & $2(5.4)$ & $0(0)$ & $0(0)$ & $0(0)$ & $0(0)$ & $0(0)$ \\
\hline Cefuroxime & $10(27)$ & $8(50)$ & $2(50)$ & $1(33.3)$ & $1(50)$ & $1(50)$ \\
\hline Amoxicilin & $33(89.2)$ & $13(81.2)$ & $3(75)$ & $3(100)$ & $2(100)$ & $2(100)$ \\
\hline Gentamycin & 7 (18.9) & $2(12.5)$ & $1(25)$ & $0(0)$ & $0(0)$ & $0(0)$ \\
\hline Cotrimoxazol & $16(43.2)$ & $13(81.2)$ & $2(50)$ & $1(33.3)$ & $0(0)$ & $2(100)$ \\
\hline Meropenem & $0(0)$ & $2(12.5)$ & $1(25)$ & $1(33.3)$ & $0(0)$ & $0(0)$ \\
\hline Cefotaxime & $12(32.4)$ & $3(18.7)$ & $1(25)$ & $2(66.7)$ & $1(50)$ & $0(0)$ \\
\hline Cloxacilin & $18(48.6)$ & $7(43.7)$ & $1(25)$ & $3(33.3)$ & $1(50)$ & $0(0)$ \\
\hline Vancomycin & $11(29.7)$ & $4(25)$ & $0(0)$ & $0(0)$ & $1(50)$ & $2(100)$ \\
\hline
\end{tabular}

Note: $\mathrm{EMCH}=$ Enam medical college \& hospital

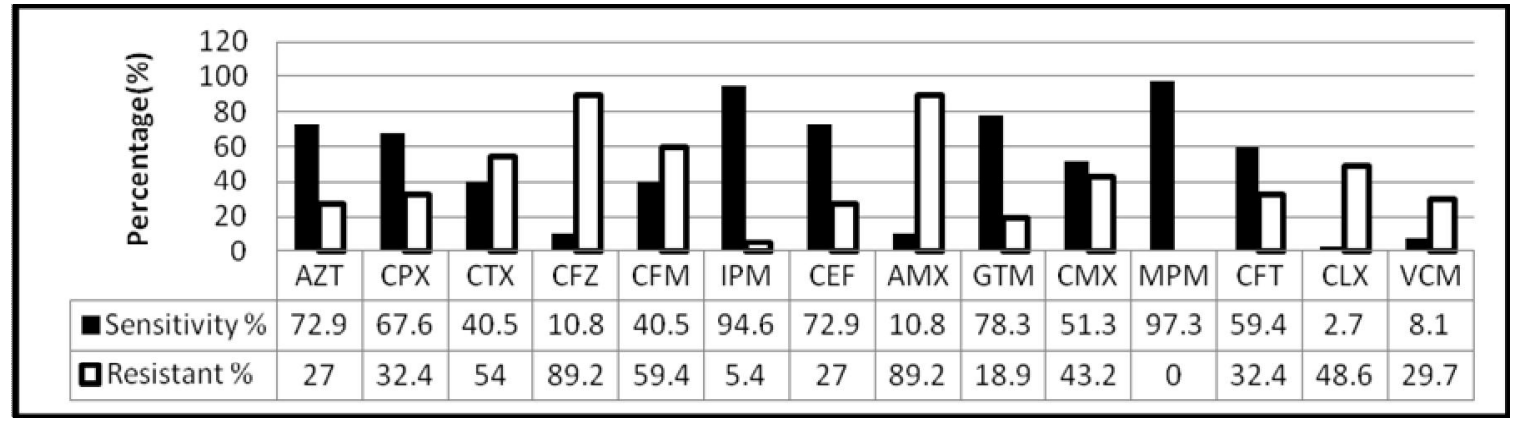

Figure 2. Patterns of antimicrobial susceptibility in $S$. aureus $(n=37)$ at Enam medical college \& hospital (EMCH) in Savar, Dhaka from July 2014-June 2015.

Different antibiotic discs used in sensitivity tests (quantity/disc) are: $($ azithromycin $=\mathrm{AZT}$, ciprofloxacin $=\mathrm{CPX}$, ceftriaxone $=$ CTX, ceftazidime $=\mathrm{CFZ}$, cefixime $=\mathrm{CFM}$, imipenem $=\mathrm{IPM}$, cefuroxime $=\mathrm{CEF}$, amoxicillin $=\mathrm{AMX}$, gentamicin $=\mathrm{GTM}$, cotrimoxazole $=\mathrm{CMX}$, meropenem $=\mathrm{MPM}$, cefotaxime $=\mathrm{CFT}$, cloxacilin $=\mathrm{CLX}$, vancomycin $=\mathrm{VCM})$ were tested by the disk diffusion method.

\section{Discussion}

The main objective of our study was to ascertain the current prevalence bacteria responsible for LRTIs among the population living in rural and suburban areas. Our study revealed the association of pathogenic bacteria in various respiratory tract infections as well as their antibiotic susceptibility profile. In this study, majority of the processed sputum samples, 64 (64\%) showed significant bacterial growth. Previous studies from different areas of Southeast Asia demonstrated an aetiological diagnosis between $45-80 \%$ (Okesola and
Ige, 2007; Panda et al., 2012). An isolation rate of 64\% was observed in pathogenic bacteria; which was similar to other previous reports (Okesola and Ige, 2007).

LRTIs were more common in males than that of females. Male prevalence of LRTI may be due to their exposure to different group of population and due to some associated risk factors of respiratory tract infection such as smoking, alcohol consumption and COPD (Panda et al., 2012). Our findings corroborated with the results of other studies accomplished by Shah et al. (2010) and Akingbade et al. (2012). Age range 
distribution of bacteria isolates showed that age group of 21-30 years found to be highly susceptible to pathogenic bacteria followed by age group of 41-50 years. From our study, it was observed that the young adults and the elderly were most at risk of a severe respiratory condition.

Among 64 bacterial isolates, six different types of bacteria were recognized. Greater number of the isolates was gram positive cocci (GPC), i.e. $89.06 \%$, and $10.93 \%$ were gram negative bacilli (GNB). Of 64 GPC in total, S. aureus (64.9\%) was the most common isolate whereas $P$. aeruginosa was only $28.5 \%$ among the gram negative bacteria. In our study, $S$. aureus $(57.81 \%)$ was the most common pathogen isolated. In previous studies $S$. pneumonia (25.0\%) was found to be the second most predominant organism which was isolated while Klebsiella pneumonia (42.85\%) to be the most predominant isolate among gram-negatives recovered from patients with LRTIs (Mishra et al., 2012; Jafari et al., 2006). Again, our results showed that in LRTI patients $P$. aeruginosa isolate was present insignificantly which differed from earlier report showing significant presence of Pseudomonas aeruginosa isolates in LRTI patients (Mishra et al., 2012; Jafari et al., 2006).

Antimicrobial susceptibility test performed on 64 bacterial isolates in the present study showed that meropenem and imipenem were the most effective (nearly $100 \%$ sensitivity) antibiotic against grampositive bacteria and amoxicillin was the least effective one (100\% resistance). Similar study conducted by Gauchan et al. (2006) and Park (2011) showed that for gram-positive bacteria, ciprofloxacin was the most effective antibiotic and the least was co-trimoxazole. $S$. aureas was mostly resistant to amoxicillin and ceftazidime, cefixime and cloxacilin. On the other hand, S. pneumoniae was found to be mostly resistant against ceftazidime and amoxicillin followed by cotrimoxazole and cefuroxime.

In case of gram negative bacteria, susceptibility pattern was found to be variable. In case of Pseudomonas spp., azithromycin, cotrimoxazole, gentamycin were the most effective (100\% sensitivity) antibiotics and amoxicillin was the least effective one (0\% sensitivity). Klebsiella spp was mostly resistant to ceftriaxone, ceftazidime and amoxicillin (100\%). E. coli was resistant to amoxicillin, cotrimoxazole and vancomycin (100\%). Antibiotic susceptibility test of the isolates from previous study showed that $100 \%$ gramnegative bacteria were sensitive to chloramphenicol but only $20.6 \%$ were to co-trimoxazole (Park, 2011).

\section{Conclusion}

LRTIs comprise a wide range of diseases from acute bronchitis to severe pneumonia leading to death. This study reveals that varieties of pathogens are responsible for LRTI and antibiotics resistance has become a great public health issue. The most of the bacteria were highly resistant to common antibiotics. The causes of the resistance may be due to irrational use and abuse of drugs, and mutation of microorganisms. Proper identification of the probable pathogens and their antibiotic susceptibility pattern can help our health professionals to choose the antibiotic therapy and improve the outcome. The possibility of reducing resistance by controlling the use of antibiotics is a reasonable approach. Inappropriate and irrational drug usage should be avoided. In our study, we showed the current scenario of resistance to commonly used antibiotics by organisms causing lower respiratory tract infections (LRTIs) in Bangladesh. It may help the government's regulatory authority to develop a policy about rational prescription of antibiotics to minimize resistance of antibiotics and also to ensure the maximum safety to the health of patients.

\section{Conflict of Interests}

The authors declare that there is no conflict of interests regarding the publication of this study.

\section{References}

Ahmed, M.S., Jakribettu, R.P., Meletath, S.K., Arya, B. and Shakir, V.P.A. 2013. Lower respiratory tract infections (LRTIs): an insight into the prevalence and the antibiogram of the gram negative respiratory, bacterial agents. J. Clin. Diag. Res. 7, 253-256.

Akingbade, O.A., Ogiogwa, J.I., Okerentugba, P.O. and Innocent-Adiele, H.C. 2012. Prevalence and antibiotic susceptibility pattern of bacterial agents involved in lower respiratory tract infections in Abeokuta, Ogun State, Nigeria. Report and Opinion. 4, 25-30. 
Bauer, A.W., Kirby, W.M.M., Sherris, J.C. and Turck, M. 1966. Antibiotic susceptibility testing by a standardized single disk method. Am. J. Clin. Pathol. 45, 493-496.

Carroll, K.C. 2002. Laboratory diagnosis of lower respiratory tract infections: controversy and conundrums. J. Clin. Microbiol. 40, 3115-3120.

Cheesbrough, M (ed). 2000. Pseudomonas and related oorganisms; biochemical test to identify bacteria; antimicrobial susceptibility testing in: district laboratory practice in tropical countries. Low price edition. New York: Cambridge University Press. pp. 1933-1943.

Clinical and Laboratory Standards Institute (CLSI. Performance Standards for Antimicrobial Susceptibility Testing. Wayne, PA: CLSI. 2012; 32: M100-S22.

Gagneja, D., Goel, N., Aggarwal, R. and Chaudhary, U. 2011. Changing trend of antimicrobial resistance among gramnegative bacilli isolated from lower respiratory tract of ICU patients: A 5-year study. Indian J. Crit. Care Med. 15, 164-167.

Gauchan, P., Lekhak, B. and Sherchan, J.B. 2006. The prevalence of lower respiratory tract infection in adults visiting Tribhuvan University Teaching Hospital. $J$. Nepal Health Res. Council 3, 23-28.

Jafari, J., Ranjbar, R., Haghi-Ashtiani, M.T., Abedini, M. and Izadi, M. 2009. Prevalence and antimicrobial susceptibility of tracheal bacterial strains isolated from paediatric patients. Pakistan J. Biolo. Sci. 12, 455-458

Kollef, M.H., Golan,Y., Micek, S.T., Shorr, A.F. and Restrepo, M.I. 2011. Appraising contemporary strategies to combat multidrug resistant Gram-negative bacterial infections-proceedings and data from the Gram-negative resistance summit. Clin. Infect Dis. 53, S33-55.

Mahmoud, A. and Balkhy, H.H. 2012. The prevalence of antimicrobial resistance in clinical isolates from Gulf Corporation Council countries. Antimicrob. Resist. Infect Contl. 1, 26-31 doi: 10.1186/2047-2994-1-26
Mishra, S.K., Kathel, H.P., Acharya, J., Shah, N.P., Shah, A.S. and Sherchand, J.B. 2012. Recent trends of bacterial etiology of LRTI in Nepal. Int. J. Infect Microbial. 1, 38.

Ndip, R.N., Ntiege, E.A., Ndip, L.M., Nkwelang, G., Aoachere, J.F. and Akenji, T.N. 2008. Antimicrobial resistance of bacterial agents of the upper respiratory tract of school children in Buea, Cameroon. J. Health Popul. Nutr. 26, 397-404.

Okesola A.O. and Ige, O.M. 2007. Trends in bacterial pathogens of lower respiratory tract infections. Indian J. Chest. Dis. Allied. Sci. 50, 270-273.

Paganin, F., Lilienthal, F., Bourdin, A., Lugagne, N., Tixier, F. and Ge'nin, R. 2004 Severe community-acquired pneumonia: assessment of microbial aetiology as mortality factor. Eur. Respir. J. 24, 779-785.

Panda, S., PremaNandini, B. and Ramani, T.V. 2012. Lower respiratory tract infection- bacteriological profile and antibiogram pattern. Int. J. Cur. Res. Rev. 4, 149-155.

Park, K. 2011. Park's Textbook of preventive and social medicine. M/S BanarsidasBhanot Publishers. $19^{\text {th }}$ ed, $p$. 91.

Shah, B.A., Singh, G., Naik, M.A. and Dhobi, G.N. 2010. Bacteriological and clinical profile of Community acquired pneumonia in hospitalized patients. Lung India 27, 54-57.

Tripathi, P.C. and Dhote, K. 2014. Lower respiratory tract infections: Current etiological trends and antibiogram. J. Pharm. Biomed. Sci. 4, 249-255.

WHO 2012. Antimicrobial resistance: no action today, no cure tomorrow, World Health Day- 7 April. 